NASA Technical Memorandum 104462

AIAA-91-1420

\title{
Scaling Analysis Applied to the NORVEX Code Development and Thermal Energy Flight Experiment
}

J. Raymond Lee Skarda, David Namkoong, and Douglas Darling Lewis Research Center

Cleveland, Ohio

Prepared for the

26th Thermophysics Conference

sponsored by the American Institute of Aeronautics and Astronautics

Honolulu, Hawaii, June 24-26, 1991

\section{N/Sก}




\title{
Scaling Analysis Applied to the NORVEX Code Development \& Thermal Energy Flight Experiment
}

\author{
J. Raymond Lee Skarda David Namkoong Douglas Darling \\ NASA Lewis Research Center \\ Cleveland, Ohio 44135
}

\begin{abstract}
$\underline{\text { Abstract }}$
A scaling analysis is used to investigate the dominant flow processes that occur in molten phase change material (PCM) under $1 \mathrm{~g}$ and microgravity conditions. Results of the scaling analysis are applied to the development of the NORVEX computer program and the preparation of the TES flight experiment. The NORVEX computer program which is being developed to predict melting and freezing with void formation in a $1 \mathrm{~g}$ or microgravity environment of the PCM is described. NORVEX predictions are compared with the scaling and similarity results. The approach to be used to validate NORVEX with TES flight data is also discussed. Similarity and scaling show that the inertial terms must be included as part of the momentum equation in either the $1 \mathrm{~g}$ or microgravity environment (a creeping flow assumption is invalid). A $10^{-4} \mathrm{~g}$ environment was found to be a suitable "microgravity" environment for the proposed PCM.
\end{abstract}

\section{Nomenclature}

Bd Dynamic Bond number

g gravitational acceleration

Gr Grashof number

L axial length

Ma Marangoni number

p pressure

Pr Prandtl number

r radial coordinate

$\mathrm{Ra}$ Rayleigh number

$T$ temperature

u velocity

z axial coordinate

$\alpha \quad$ thermal diffusivity

$\beta \quad$ thermal expansion coefficient

$\mu \quad$ kinematic viscosity

$\nu \quad$ dynamic viscosity

$\rho$ density

$\sigma \quad$ surface tension $\underline{\text { Introduction }}$

Various Fluoride salts (e.g. $\mathrm{LiF}, \mathrm{LiF}-\mathrm{CaF}_{2}, \mathrm{NaF}$ ) have been identified as candidate thermal energy storage (TES) media for space solar dynamic power systems. ${ }^{1}$ The Fluoride salts have high heats of fusion at melting temperatures that provide a constant temperature heat source for proposed Brayton or Stirling cycle heat engines. The use of phase change materials (PCMs) have been shown to translate into overall receiver weight savings. ${ }^{1,2}$ However, Fluoride salts exhibit as much as a $30 \%$ change in volume during the phase change process. This leads to the formation of a void(s) during the solidification process.

Two concerns which emerge as a result of void formation are the potential for "ratcheting" of the TES canister wall and the development of "hot spots" on TES canister. Ratcheting is the distortion that could occur if the liquid PCM is unable to expand into the void and instead creates stresses on the container wall as PCM continues to melt. Hot spots occur at locations where voids are present between the wall and the PCM. The present lack of understanding of the freeze-thaw process, coupled with large volume changes in a microgravity environment results in conservatively designed TES/receiver systems.

Microgravity experience and data do not currently exist with thermal energy storage materials. In fact numerical values of gravitational acceleration that designate a "microgravity" environment for TES materials also appear nonexistent in current literature. Modeling capabilities to predict void nucleation, movement, and final location/shape are only now being developed. Ground test data of TES subassemblies and components are beginning to emerge. ${ }^{3,4,5}$ A computational effort, the NORVEX (NASA/Oak Ridge Void Experiment) computer program development, and an experimental effort, the Thermal Energy Storage Flight Experiment, have also been initiated to address the behavior of thermal energy storage materials, particularly void shape and location in a microgravity environment. ${ }^{1}$ NORVEX 
was developed in the absence of any existing

3-dimensional computer program that could describe the PCM phase change behavior which includes void formation. Results from the TES Flight Experiment will provide fundamental information concerning void behavior and will also be used to validate the NORVEX code.

The use of scaling analyses and relevant dimensionless (similarity) groups provides an understanding of the physical processes that will prove valuable during the development of the numerical models or physical experiments. Identification of the dominant processes that are not obvious from a complex numerical analysis may be possible using scaling results. This may justify neglecting (or retaining) certain terms in the governing equations and boundary conditions during the development of numerical models. Scaling and similarity can provide guidance during the preparation of a TES experiment by addressing critical questions such as the largest allowable gravitational acceleration that constitutes a microgravity environment. At a minimum similarity results yield logical first estimates of boundary and operating conditions when the necessary analytical or computational tools are not available. Scaling and similarity results of this nature do not exist in the current microgravity TES literature.

The work described herein is part of the Advance Solar Dynamic Receiver program at the NASA Lewis Research Center. The objectives of the work presented in this paper are:

- Apply relevant dimensionless parameters and a scaling analysis to characterize the $1 \mathrm{~g}$ and microgravity molten PCM behavior and to study the necessity of various terms in the governing equations.

- Describe the capabilities of the NORVEX computer program and present NORVEX predictions for comparison with similarity and scaling results.

- Describe the approach that will be used to evaluate the NORVEX computer program with the TES flight experiment results.

\section{Thermal Configuration}

Various TES design and operating configurations have been proposed. ${ }^{1,4}$ The configuration used in the TES flight experiment and current NORVEX modeling effort is shown in Figures 1. This TES configuration will be considered further in this paper. The phase change material (PCM) is contained in a torus-shaped canister. The principal thermal feature is heat (solar flux) being input to the PCM from the outer TES canister radius, while heat is extracted via a heat pipe at the inner canister radius. The actual shape of the void is expected to be driven primarily by thermal considerations. That is, the void will form near the hotter surfaces. Therefore, the void should form near the outer wall during solidification. Buoyancy, surface tension, and wetting may also play roles in the void shape and location. In addition to hot spots, large thermal gradients may occur in the outer canister wall while heating wall regions where both the PCM and void contact the wall.

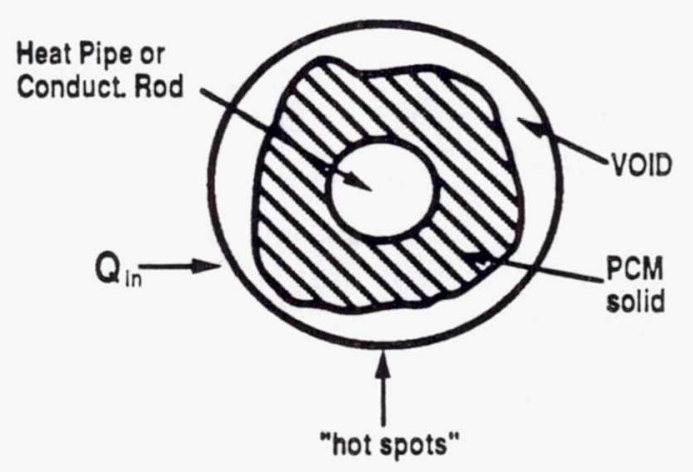

Figure 1 Thermal \& Void Configuration

\section{Scaling}

The treatment of the PCM freeze/thaw behavior including void formation and the molten PCM hydrodynamics is a complex problem to model. Without simplification and isolation of a specific set of processes, a scaling analysis rapidly becomes intractable. This scaling analysis is confined to the hydrodynamic processes of the molten PCM for the two following reasons. First is that we wish to apply some simple quantitative criteria that identifies the type of flow expected for different conditions and orientations. Application of the appropriate dimensionless groups also provides quantitative estimates of the conditions necessary to obtain the flow types and dominant processes. The second reason for scaling the hydrodynamic processes is that numerical modeling of these processes is the most computationally intensive aspect of simulating the PCM behavior. Approximately $40 \%$ of the cpu time is devoted to solving the momentum equations during typical NORVEX simulations. 
Dimensionless parameters commonly used to characterize surface tension or buoyancy dominated flows are defined in Table 1 . The proposed geometry, void orientation, and characteristic lengths are shown in Figures $2 a \&$ b. The appropriate characteristic length generally depends on the orientation of the dominant driving force. However in the absence of a scaling analysis to a clearly defined problem, some ambiguity remains as observed in the calculation of $\mathrm{Ra}$ and Gr. While the characteristic length for computing $\mathrm{Ra}$ and $\mathrm{Gr}$ is generally chosen perpendicular to the direction of gravity for buoyancy driven flows in an enclosure, a characteristic length in the direction of gravity is also used. ${ }^{6,7}$ Using property values from Table 2 , values of the dimensionless groups are given in Table 3 for different values of $\Delta T$, $\mathrm{g}$, and characteristic lengths.

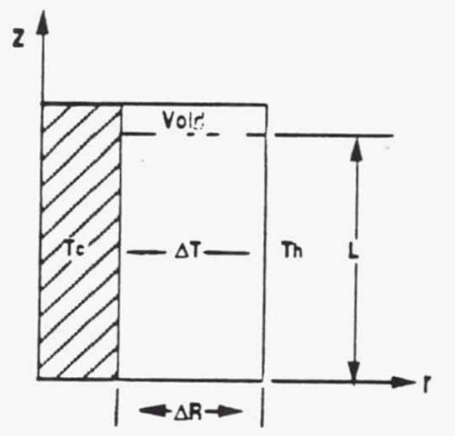

Figure 2a Free Surface Perpendicular To $\mathrm{g}_{\mathrm{z}}$

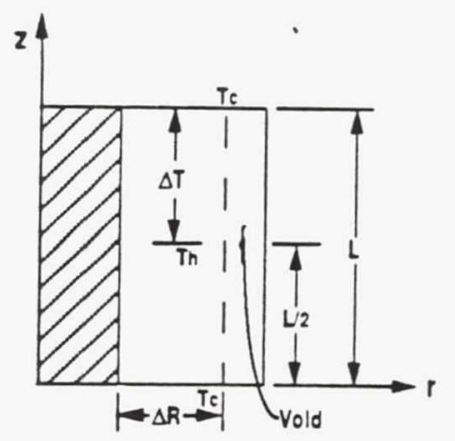

Figure 2b Free Surface Parallel To $g_{2}$

All $1 \mathrm{~g}$ dynamic Bond number terms, $\mathrm{Bd} \beta \Delta \mathrm{t}$, in Table 3 are greater than 10 while all $10^{-4} \mathrm{~g}$ Bond Number terms are less than 0.05 . We conclude that buoyancy forces will dominate over the surface tension forces in $1 \mathrm{~g}$ and the reverse is true in a microgravity environment, $10^{-4} \mathrm{~g}$. The results corroborate the expectations expressed in the microgravity TES literature. ${ }^{4,8}$ However, the $\mathrm{Bd}$ values may not be that much greater or less than 1 to completely neglect the less dominant force. This must be established from numerical or experimental results.

Table 1 Definition of Dimensionless Groups

$\begin{array}{lll}\text { Ar } & \text { Aspect Ratio } & \frac{\Delta R}{L} \\ \operatorname{Pr} & \frac{\text { momentum diffusivity }}{\text { thermal diffusivity }} & \frac{v}{\alpha} \\ G r_{L \varepsilon} & \frac{\text { buoyancy forces }}{\text { viscous forces }} & \frac{\beta g \Delta T L_{c}^{3}}{\nu^{2}} \\ R a_{L \varepsilon} & \frac{\text { buoyant transprt of energy }}{\text { conductn transprt of energy }} & \frac{\beta g \Delta T L_{c}^{3}}{\nu \alpha} \\ M a_{L E} & \frac{\text { surfce tension trnsprt of energy }}{\text { condution transprt of emergy }} & \frac{(\partial \sigma / \partial T) \Delta T L_{c}}{\mu \alpha} \\ B d \beta \Delta T & \frac{B u o y a n c y \text { force }}{\text { surface tension force }} & \frac{\beta P g L_{c}^{2}}{(\partial \sigma / \partial T)}\end{array}$

Table 2 LiF Properties $(T=1140 \mathrm{~K})$

$\begin{array}{llll}\rho & 1.79 \dot{\mathrm{g}} / \mathrm{cm}^{3} & \beta & 2.73 \mathrm{e}-4 \mathrm{~K}^{-1} \\ \alpha & 3.94 \times 10-3 \mathrm{~cm}^{2} / \mathrm{s} & \sigma & 233.9 \text { dynes } / \mathrm{cm} \\ \mu & 0.0221 \mathrm{~g} / \mathrm{cm}^{2} \mathrm{~s} & -\mathrm{d} \sigma / \mathrm{dT} & 0.0988 \mathrm{~g} / \mathrm{s}^{2}-\mathrm{K} \\ \nu & 0.0123 \mathrm{~cm}^{2} / \mathrm{s} & \mathrm{g} & 981 \mathrm{~cm} / \mathrm{s}\end{array}$

Table 3 Values of Dimensionless Groups

\begin{tabular}{lllll} 
& \multicolumn{2}{c}{$\Delta \mathrm{T}=50 \mathrm{~K}$} & \multicolumn{2}{c}{$\Delta \mathrm{T}=10 \mathrm{~K}$} \\
& \multicolumn{1}{c}{$1 \mathrm{~g}$} & $10-4 \mathrm{~g}$ & $1 \mathrm{~g}$ & $10-4 \mathrm{~g}$ \\
$\mathrm{Ar}$ & 0.229 & 0.229 & 0.229 & 0.229 \\
$\mathrm{Pr}$ & 3.13 & 3.13 & 3.13 & 3.130 \\
$\mathrm{Gr}$ & $2.90 \times 10^{7}$ & 2900 & $5.79 \times 10^{6}$ & 579 \\
$\mathrm{Gr}$ & $3.50 \times 10^{5}$ & 35.0 & $6.99 \times 10^{4}$ & 6.99 \\
$\mathrm{Ra}_{\mathrm{L}}$ & $4.91 \times 10^{7}$ & 4910 & $9.82 \times 10^{6}$ & 982 \\
$\mathrm{Ra}_{\Delta \mathrm{R}}$ & $1.09 \times 10^{6}$ & 109 & $2.19 \times 10^{5}$ & 21.9 \\
$\mathrm{Ma}_{\Delta \mathrm{R}}$ & $8.95 \times 10^{4}$ & $8.95 \times 10^{4}$ & $1.79 \times 10^{4}$ & $1.79 \times 10^{4}$ \\
$\mathrm{BdL}^{\mathrm{a}}$ & 232 & 0.0232 & 232 & 0.0232 \\
$\mathrm{BdR}^{\mathrm{b}}$ & 12.1 & $1.21 \times 10^{-3}$ & 12.1 & $1.21 \times 10^{-3}$ \\
\hline $\mathrm{L}=6.89 \mathrm{~cm}$ & ${ }^{\mathrm{a}} \mathrm{BdL} \equiv \mathrm{Bd}_{\llcorner} \beta \Delta \mathrm{T}$ \\
$\Delta \mathrm{R}=1.58 \mathrm{~cm}$ & ${ }^{\mathrm{b}} \mathrm{BdR} \equiv \mathrm{Bd}_{\Delta \mathrm{R}} \beta \Delta \mathrm{T}$ & \\
\multicolumn{5}{c}{}
\end{tabular}


For flows in a 1-g environment, $\mathrm{Ra}>10^{5}$, therefore, heat transport by convection must be included in the modeling of the liquid PCM. None of the Ra values exceeds $10^{8}$ which suggests the flow is laminar ${ }^{6}$. For the given conditions, turbulence does not need to be considered during the development of a numerical model.

The free surface orientation shown in Figure 2a, suggests that the $\mathrm{L}_{c}$ is in the axial direction. However, the surface tension driven flow is not likely to extend the full distance, $L$. This assumption is supported by a $0-\mathrm{g}$ surface tension driven flow analysis in a rectangular enclosure $(\mathrm{Ar}=1 / 3){ }^{9}$ The free surface orientation is that of Figure 2a. A flow cell is observed in the upper region of the enclosure, $\mathrm{L}_{c} \simeq \mathrm{L} / 2$, while a quiescent region exists below. ${ }^{9}$ A Marangoni number based on $\mathrm{L}$ loses meaning since $\mathrm{L}$ is not a reasonable characteristic length for the Figure $2 \mathrm{a}$ configuration. Numerical or experimental analyses may be required to determine the extent of surface tension driven flows that develop in a Figure $2 \mathrm{a} . \mathrm{Ma}_{\Delta \mathrm{R}}$ is relevant to free surface orientation shown in Figure $2 \mathrm{~b}$. For the $10^{-4} \mathrm{~g}$ results in Table 3, $\mathrm{Ma}_{\Delta \mathrm{R}}>10^{4}$ which indicates that heat transport by the surface tension driven convection must be not be neglected when developing fluid dynamic model.

A more rigorous identification of the dominant terms in the governing equations and boundary conditions is obtained from a scaling analysis. However, an initial guess of the dominant driving force is required to choose the appropriate reference parameters. We shall assume that the dominant driving force is surface tension and that the free surface is oriented as shown in Figure $2 \mathrm{~b}$. These assumptions restrict the process to a microgravity environment since Table 3 results indicate that buoyancy forces dominate under $1-\mathrm{g}$ conditions and the void orientation of Figure $2 \mathrm{~b}$ is not plausible in a 1-g environment. For the configuration shown in Figure 2b, the surface tension force is parallel to the direction of gravity. The scaling approach presented by Ostrach ${ }^{10}$ is applied to the current analysis.

The following conditions and assumptions are applied to define the problem.

The temperature gradient occurs on the free surface from the interface center at $\mathrm{L} / 2$ to either end, $\mathrm{z}=0$ and $\mathrm{z}=\mathrm{L}$. The hot temperature, $\mathrm{T}_{\mathrm{h}}$, exists at $\mathrm{L} / 2$ while the cold temperature, $T_{c}$, exists at $\mathrm{z}=0$ and $\mathrm{z}=\mathrm{L}$. The $\Delta \mathrm{T}, \mathrm{T}_{\mathrm{h}}-\mathrm{T}_{\mathrm{c}}$, is assumed to be $10 \mathrm{~K}$. Additional assumptions are: incompressible flow, steady state, flat liquid/void interface (in $\mathrm{z}$ direction), completely molten PCM, negligible endwall wetting, $10^{-4} \mathrm{~g}$. The resulting governing equations and free surface boundary condition: continuity, r-momentum, zmomentum, energy, free surface boundary condition are given by equations 1-5.

$$
\begin{aligned}
& 1 / \mathrm{r} \frac{\partial\left(\mathrm{ru}_{\mathrm{r}}\right)}{\partial \mathrm{r}}+\frac{\partial \mathrm{u}_{\mathrm{z}}}{\partial \mathrm{z}}=0 \\
& \mathrm{u}_{\mathrm{r}} \frac{\partial \mathrm{u}_{\mathrm{r}}}{\partial \mathrm{r}}+\mathrm{u}_{\mathrm{z}} \frac{\partial \mathrm{u}_{\mathrm{r}}}{\partial \mathrm{z}}=\cdot \frac{1}{\rho} \frac{\partial \mathrm{p}}{\partial \mathrm{r}}+\beta \mathrm{g}_{\mathrm{r}}\left(\mathrm{T}-\mathrm{T}_{\mathrm{c}}\right) \\
& +\mathrm{v}\left[\frac{\partial}{\partial \mathrm{r}}\left(1 / \mathrm{r} \frac{\partial\left(\mathrm{ru}_{\mathrm{r}}\right)}{\partial \mathrm{r}}\right)+\frac{\partial^{2}\left(\mathrm{u}_{\mathrm{r}}\right)}{\partial \mathrm{z}^{2}}\right] \\
& \mathrm{u}_{\mathrm{r}} \frac{\partial \mathrm{u}_{\mathrm{z}}}{\partial \mathrm{r}}+\mathrm{u}_{\mathrm{z}} \frac{\partial \mathrm{u}_{\mathrm{z}}}{\partial \mathrm{z}}=\cdot \frac{1}{\mathrm{\rho}} \frac{\partial \mathrm{p}}{\partial \mathrm{r}} \\
& +\mathrm{v}\left[1 / \mathrm{r} \frac{\partial}{\partial \mathrm{r}}\left(\mathrm{r} \frac{\partial\left(\mathrm{u}_{\mathrm{z}}\right)}{\partial \mathrm{r}}\right)+\frac{\partial^{2}\left(\mathrm{u}_{\mathrm{z}}\right)}{\partial \mathrm{z}^{2}}\right] \\
& u_{\mathrm{T}} \frac{\partial \mathrm{T}}{\partial \mathrm{r}}+\mathrm{u}_{\mathrm{z}} \frac{\partial \mathrm{T}}{\partial \mathrm{z}}=\alpha\left[1 / \mathrm{r}\left(\frac{\partial}{\partial \mathrm{r}}\left(\mathrm{r} \frac{\partial \mathrm{T}}{\partial \mathrm{r}}\right)+\frac{\partial^{2} \mathrm{~T}}{\partial \mathrm{z}^{2}}\right]\right. \\
& -\mu \frac{\partial \mathrm{u}_{\mathrm{z}}}{\partial \mathrm{r}}=\left(\frac{\partial \sigma}{\partial \mathrm{T}}\right)\left(\frac{\partial \mathrm{T}}{\partial \mathrm{z}}\right)
\end{aligned}
$$

The variables defined to nondimensionalize the governing equations and boundary conditions are:

$$
\begin{aligned}
& \mathrm{z}^{*}=\mathrm{z} / \mathrm{L}, \quad \mathrm{r}^{*}=\mathrm{r} / \mathrm{r}_{\mathrm{c}}, \quad \mathrm{u}_{\mathrm{z}}^{*}=\mathrm{u}_{\mathrm{z}} / \mathrm{U}_{\mathrm{R}}, \\
& \mathrm{u}_{\mathrm{r}}^{*}=\mathrm{u}_{\mathrm{R}} / \mathrm{U}_{\mathrm{R}}\left(\mathrm{r} / \mathrm{r}_{\mathrm{c}}\right), \quad \mathrm{p}=\mathrm{p} / \rho \mathrm{U}_{\mathrm{R}}{ }^{2}, \\
& \Delta \mathrm{T}=\mathrm{Tw}-\mathrm{Tc}, \quad \boldsymbol{\theta}=\left(\mathrm{T}-\mathrm{T}_{\mathrm{c}}\right) / \Delta \mathrm{T}
\end{aligned}
$$

The appropriate forms of both the characteristic length, $\mathrm{r}_{\mathrm{c}}$, and the reference velocity, $\mathrm{U}_{\mathrm{R}}$, are determined by the magnitude of the surface tension Reynolds Number, $\mathrm{Re}_{f}$, and the aspect ratio, Ar. According to Ostrach ${ }^{10}$ the appropriate $r_{c}$ is $\Delta R$ for viscous type flow which exists for $A r^{2} \mathrm{Re}_{\sigma}<<1$. For $\mathrm{Ar}^{2} \mathrm{Re}_{\sigma}>>1$, a boundary layer type flow occurs and the appropriate $r_{c}$ is the boundary layer thickness, $\delta$. $\mathrm{U}_{\mathrm{R}}$, is obtained from nondimensionalization of the free surface boundary condition, equation $5 .{ }^{10}$ To initially determine the type of flow, $U_{R}$ and $R e$ are computed from equations 6 and 7, which assume a viscous type flow. If a boundary layer flow occurs, then $U_{R}$ is rederived using $r_{c}=\delta$ in the 
nondimensionalization of equation 5.

$$
\begin{aligned}
& \mathrm{U}_{\mathrm{R} \sigma}=(\partial \sigma / \partial \mathrm{T}) \Delta \mathrm{T} \Delta \mathrm{R} / \mu \mathrm{L} \\
& \mathrm{Re}_{\sigma}=\mathrm{U}_{\mathrm{R}_{\sigma}} \mathrm{L}_{\mathrm{c}} / \nu
\end{aligned}
$$

Using the properties given in Table $2, \mathrm{Ar}^{2} \mathrm{Re}_{\sigma}=1208$. Therefore a boundary layer exists and the appropriate $r_{c}$ is boundary layer thickness, $\delta$. Since $\delta / L$ is proportional to $1 / \operatorname{Re}^{1 / 2}$ for boundary layer flows, $\delta$ can be written in terms of $\mathrm{L} / \mathrm{Re}^{1 / 2}$. The resulting expressions for $r^{*}, u^{*}$ and $U_{R}$ are given below. The subscript bl emphasizes that $U_{R}$ is defined for a boundary layer type flow.

$$
\begin{gathered}
r^{*}=r / \delta=r / L_{c}(R e)^{1 / 2} \quad u^{*}=u_{R} / U_{R}(R e)^{1 / 2} \\
U_{R b l}=\left[(\partial \sigma / \partial T)^{2} \Delta T^{2} \nu / \mu^{2} L\right]^{1 / 3}
\end{gathered}
$$

The nondimensional governing equations are given by equations 8 through 10 , respectively.

$$
\begin{aligned}
& \mathrm{u}_{\mathrm{r}} * \frac{\partial \mathrm{u}_{\mathrm{r}}^{*}}{\partial \mathrm{r}} * \mathrm{u}_{\mathrm{z}}^{*} \frac{\partial \mathrm{u}_{\mathrm{r}}^{*}}{\partial \mathrm{z}^{*}}=-\operatorname{Re}_{\mathrm{L}} \frac{\partial \mathrm{p}}{\partial \mathrm{r}} * \\
& +\frac{\partial}{\partial r}\left(1 / \mathrm{r} * \frac{\partial\left(\mathrm{r}^{*} \mathrm{u}_{\mathrm{r}}^{*}\right)}{\partial \mathrm{r}^{*}}\right)+1 / \operatorname{Re}_{\mathrm{L}} \frac{\partial^{2}\left(\mathrm{u}_{\mathrm{r}}^{*}\right)}{\partial \mathrm{z}^{* 2}} \\
& u_{r}^{*} \frac{\partial u_{z}^{*}}{\partial r^{*}}+u_{z^{*}} \frac{\partial u_{z}^{*}}{\partial z^{*}}=-\frac{\partial p}{\partial z}{ }^{*}+\frac{G_{L} r_{c}}{R e^{2}} \theta \\
& +1 / \mathrm{r} * \frac{\partial}{\partial \mathrm{r}^{*}}\left(\frac{\mathrm{r} * \partial \mathrm{u}_{\mathrm{Z}}^{*}}{\partial \mathrm{r}^{*}}\right)+1 / \operatorname{Re}_{\mathrm{L}} \frac{\partial^{2}\left(\mathrm{u}_{\mathrm{z}}^{*}\right)}{\partial \mathrm{z}^{* 2}} \\
& u_{r_{r}} * \frac{\partial \theta}{\partial r}+u_{z}^{*} \frac{\partial \theta}{\partial z}=\frac{1}{\operatorname{Prr}^{*} *} \frac{\partial}{\partial r^{*}}\left(\frac{r * \partial \theta}{\partial r^{*}}\right) \\
& +\cdot \frac{1}{\operatorname{Pr} \operatorname{Re}} \frac{\partial^{2} \theta}{\partial z^{* 2}}
\end{aligned}
$$

Using the property data in Table 2, the values for the dimensionless coefficients found in equations 5-7 are tabulated in Table 4.

Results from the scaling analysis suggest that a boundary type flow will develop in the molten PCM. Furthermore, a radial liquid thickness, $\Delta \mathrm{R}$ must be less than $0.7 \mathrm{~cm}$ for a viscous type flow $\left(A r^{2} \mathrm{Re}_{\sigma}<0.1\right)$ to exist in the molten PCM. Therefore, a boundary layer flow is expected in a microgravity environment during most of the melting or freezing process. Even under microgravity conditions, a stokes flow assumption is generally invalid and the inertial terms must be included as part of the momentum equations. Based on Table 4 results, the axial viscous terms in equations $8 \& 9$, the buoyancy term, and the axial conduction term can be neglected in the proposed microgravity configuration. If solidification occurs uniformly in the radial direction these terms can be neglected for molten regions with a radial thickness greater than $0.08 \mathrm{~cm}$.

\section{Table 4 Values of Dimensionless Groups Derived From Scaling}

$\begin{array}{llll}\mathrm{Re}_{\mathrm{bl}} & 539 & 1 / \mathrm{Re}_{\mathrm{bl}} & 0.0019 \\ \mathrm{Pr} & 3.130 & \mathrm{Gr}_{\mathrm{LC}} / \mathrm{Re}_{\mathrm{bl}}{ }^{2} & 4.63 \times 10^{-4} \\ 1 / \mathrm{Pr} & 0.319 & 1 / \mathrm{PrRe}_{\mathrm{bl}} & 1.276 \times 10^{-4}\end{array}$

\section{$\underline{\text { Numerical Model Description }}$}

The results from the dimensionless groups and the scaling analysis indicate a numerical model should include the following effects to represent the PCM behavior in both $1-\mathrm{g}$ and $0-\mathrm{g}$ environments.

Convection and conduction effects must be included in the energy equation. The inertial, viscous, pressure, and buoyancy effects must be included in the momentum equation. The tangential surface tension gradient and viscous force balance, as well as the normal pressure and surface tension force balance need to be included in the free surface boundary equation. In this section we will look at such a model, developed to predict the behavior of the phase change material (PCM).

The NORVEX computer program has been developed to predict the behavior of the phase change material. General features of the code are summarized in Table 5. An overview of the computer program's numerical approach and capabilities is given below. An in-depth treatment of the computational approach used in NORVEX is given in Drake; the radiative transport through the LiF (PCM) is described in Williams ${ }^{12}$; and the operational requirements, input, and output descriptions are given in the NORVEX User Manual $^{13}$. In addition to PCM temperature, void movement, and void size during melting and solidification, the code predicts temperatures in the radiator flare, conductor rod, and PCM canister walls. (The radiator flare and conductor rod are components 
of the TES flight experiment described in the next section.) Once the temperature profiles of the PCM canister walls are predicted, they can be used to determine canister stresses.

The overall sequence of computations in NORVEX is shown in Figure 3. The code begins with a specified initial condition, then marches in time, simulating the PCM behavior. During a typical time-step, the energy equations are solved first, yielding the temperature of the PCM material as well as the temperatures of the radiator flare, conductor rod, and PCM canister. Then, the new void volume is determined based on the amount of PCM that changed phase during the time-step. Next, the momentum equations are solved to determine the velocities in the liquid portion of the PCM and the forces influencing void movement.

Finally, the new void location is determined using the results of the momentum and continuity equations.

\section{Table 5 NORVEX Program Features}

\begin{tabular}{|c|c|}
\hline Time Steps & Implicit/Explicit \\
\hline Geometry & $R-\Theta-Z$ \\
\hline Gravity & $\begin{array}{l}0 \leq \mathrm{g}<\infty \\
\text { arbitrary direction }\end{array}$ \\
\hline Canister Ht Flx & Input $\mathrm{q}(\mathrm{t}, \mathrm{z}, \boldsymbol{\theta})$ \\
\hline Void Interface & $\begin{array}{l}\text { Tangl Stress Bal. (Marangoni) } \\
\text { Norml Stress Bal. (Curvature) } \\
\text { Vol of Fluid Appr. }\end{array}$ \\
\hline Melt Front & $\begin{array}{l}\text { Enthalpy Method (Energy) } \\
\text { Porosity Apprch (Momentum) }\end{array}$ \\
\hline Radiant H.T. & $\begin{array}{l}\text { Transparent } \lambda<5.5 \mu \mathrm{m} \\
\text { Strong Abs } \lambda>5.5 \mu \mathrm{m}\end{array}$ \\
\hline Void H.T. & Effective Conduct \\
\hline Stress Analys & NASTRAN/ADINA \\
\hline
\end{tabular}

The temperatures in the radiator flare, conductor rod and PCM canister are determined using a finitedifferenced, unsteady conduction equation. The temperature and time dependent quantities are weighted by a user-specified degree of implicitness.

The temperatures of the PCM are determined by solving a finite-differenced energy equation. The energy equation is formulated in terms of enthalpy. Constitutive relations between enthalpy and temperature are used to determine temperature. The energy equation (in terms of enthalpy) for the PCM is solved using a predictor-corrector scheme. Velocities from the old time step are used to solve the energy equation, since the energy equation is solved before the momentum equation. But, temperature and time dependent quantities are again weighted by the degree of implicitness.

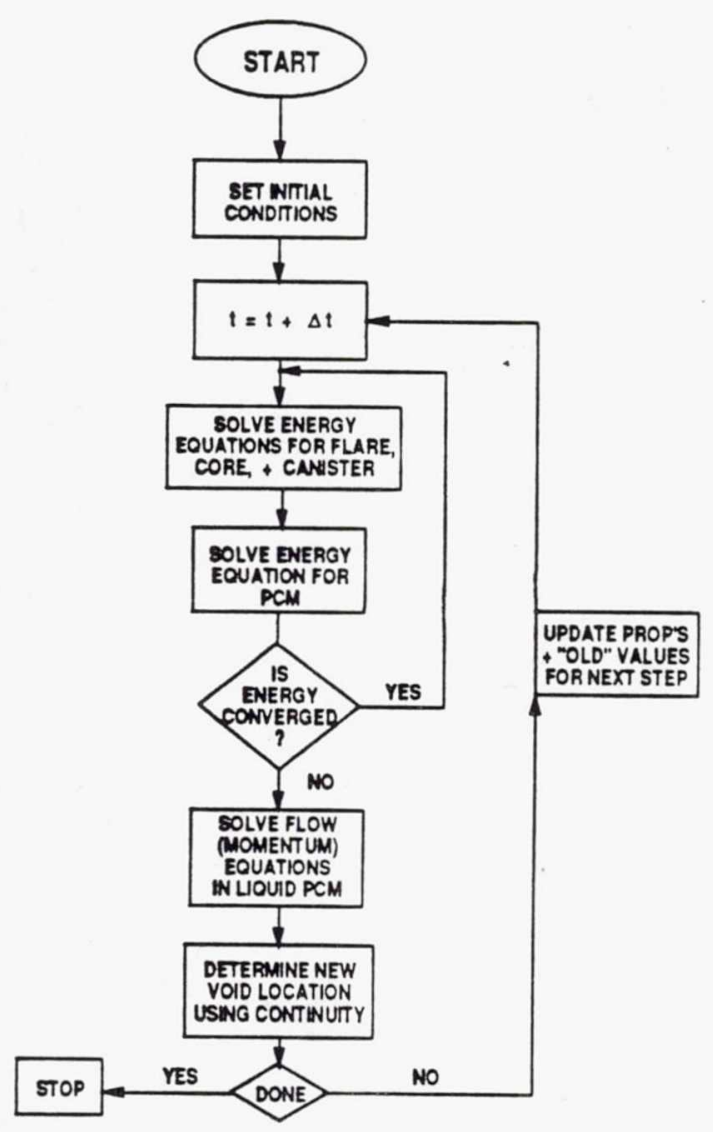

Figure 3 NORVEX Computational Procedure

After solving the energy equation, the void volume is updated based on the amount of fluid changing phase during the current time-step. A state array (fluid fraction) is used to determine if a cell is full, empty, or contains the free surface. Then, the location of the free surface is identified and the curvature of the surface computed. The determination of the free surface is actually established by computing the gradient of the fluid fraction. Tangential and normal surface forces are applied in the momentum equations of cells containing the free surface. This free surface approach is similar to the volume of fluid approach (VOF) developed by Hirt and Nichols ${ }^{14}$.

The momentum equations are solved in two parts. First, convective terms are grouped with viscous forces, buoyancy forces, and Marangoni forces. The velocity field is estimated using these terms. The rest of the momentum equation, i.e. the pressure terms and normal surface tension terms, are solved 
simultaneously with continuity to determine the pressure distribution and a corrected velocity field.

A permeability term, $K\left(x_{1}\right) U^{n+1}$, was added to the momentum equation where porosity function, $\mathrm{K}$, is some function of the liquid mass fraction, $x_{1}$. The $n+1$ superscript emphasizes that the term is applied to the $\mathbf{U}$ value at the new time-step. $\mathbf{U}$ at the new time step is used for stability, since the absolute value of $\mathrm{K}$ grows very large when driving the velocity to zero in the solid. The general form of the function, $\mathrm{K}$, is given by equation 11 . The variables, $b$ and $j_{b i g}$ are user specified constants that control how fast flow is dampened in mushy cells. Typical values of $b$ are 1 or 2 while $\mathrm{j}_{\mathrm{big}}$ is a sufficiently large value, ie $10^{5}$, that yields negligible velocities in the solid.

$$
\mathrm{K}=-\left(1-\mathrm{x}_{1}\right)^{\mathrm{b}} \mathrm{j}_{\mathrm{big}}
$$

As the a liquid phase is approached, porosity function, $\mathbf{K}\left(\mathrm{x}_{1}\right)$ approaches zero; the permeability term becomes negligible for liquid cells (nodes). As a solid state of the PCM is approached, the porosity term forces the velocities to zero in the solid cells. In nodes or cells undergoing phase change, the mushy zone, $\mathrm{k}$ becomes large and the momentum equation reduces to Darcy's Law.

Finally the new void location is determined from the results of the momentum and continuity equations. The change of PCM density during the time-step is determined from the $\operatorname{div}(\rho \mathbf{U})$. The fluid fraction of each node is then computed as $\rho_{\text {new }} /\left(x_{1} \rho+\left(1-x_{1}\right) \rho_{s}\right)$. The new free surface can then be determined from the gradient of the fluid fraction.

The principal attractiveness of the enthalpy approach for the energy equation and porosity approach for the momentum equation, is the computational domain remains the same regardless of liquid/solid fractions of the PCM in the container. The location of the solid/liquid front is simply determined by the cell liquid mass fraction.

\section{Numerical Results}

The results from NORVEX simulations are presented below. The effect of buoyancy driven convection on the melting and solidification of the PCM was investigated. Specifically, the position of the phase change front, melting times, and freezing times of a microgravity $\left(10^{-4} \mathrm{~g}\right)$ environme it were compared to $1 \mathrm{~g}$ and $0 \mathrm{~g}$ results. The PCM canister was assumed to be completely filled and no density change occurs between the solid and liquid. Since voids and free surfaces will not form under these assumptions, the only driving force for convection is buoyancy. Consequently, heat transport for the $0 \mathrm{~g}$ simulations was by conduction only.

The inner dimensions of the PCM canister were; an inner radius of $1.9 \mathrm{~cm}$, an outer radius of $3.48 \mathrm{~cm}$, and a height of $6.89 \mathrm{~cm}$. The canister wall thickness was $0.1 \mathrm{~cm}$ on all sides. During melting, heat was applied to the outer radius of the canister at a rate of $260 \mathrm{~W}$ $\left(1.63 \mathrm{~W} / \mathrm{cm}^{2}\right)$, all other walls of the canister were insulated, and the initial temperature was $1000 \mathrm{~K}$. During solidification, heat was removed from the inner radius of the canister at a rate of $454 \mathrm{~W}$ (5.36 $\mathrm{W} / \mathrm{cm}^{2}$ ), all other walls of the canister were insulated, and the initial temperature was $1200 \mathrm{~K}$. PCM properties are provided in Table 2 while additional property and geometry information are given in Skarda ${ }^{15}$. Both melting and solidification simulations were performed for gravitational accelerations of $1 \mathrm{~g}$, $10^{-4} \mathrm{~g}$, and $0 \mathrm{~g}$ acting in the $-\mathrm{z}$ direction.

Figure 4 shows the total liquid percent of the PCM (by mass) versus time for the melting simulations. As expected, the PCM melted faster in the $1 \mathrm{~g}$ environment than under microgravity or $0 \mathrm{~g}$ conditions. The melting time for the $1 \mathrm{~g}$ case is $2 \%$ less than the melting time for the $0 \mathrm{~g}$ and $10^{-4} \mathrm{~g}$ simulations. The $0 \mathrm{~g}$ and $10^{-4} \mathrm{~g}$ curves are essentially identical as shown in Figure 4. Average temperatures for the outer canister wall are $1205 \mathrm{~K}, 1267 \mathrm{~K}$, and $1268 \mathrm{~K}$ for the $1 \mathrm{~g}, 10^{-4} \mathrm{~g}$ and $0 \mathrm{~g}$, respectively. Therefore, the canister outer wall average temperature for the $1 \mathrm{~g}$ case is $53 \mathrm{~K}(4 \%)$ less than that of the $0 \mathrm{~g}$ simulation.

Figure 5a shows the progress of the melting front for the $1 \mathrm{~g}$ melting case, while figure $5 \mathrm{~b}$ shows the melting front progress for the $10^{-4} \mathrm{~g}$ and $0 \mathrm{~g}$ cases. The position of the fronts for the $10^{-4} \mathrm{~g}$ and $0 \mathrm{~g}$ cases are represented by the same set of curves since the fronts are essentially the same. The maximum difference of the melt front locations between the $10^{-4} \mathrm{~g}$ and $0 \mathrm{~g}$ cases is $0.008 \mathrm{~cm}$. which is small compared with the PCM thickness $(\Delta r)$ of $1.58 \mathrm{~cm}$. Figure $5 \mathrm{~b}$ also reveals that conduction along the top and bottom canister walls also influences the melt front orientation. The $1 \mathrm{~g}$ melt fronts are observed in Figure 5a to penetrate further near the top of canister due to the buoyancy induced flow. The hotter fluid contacts the solid PCM near the top of the canister and then cools as it falls 
along the solid/liquid interface.

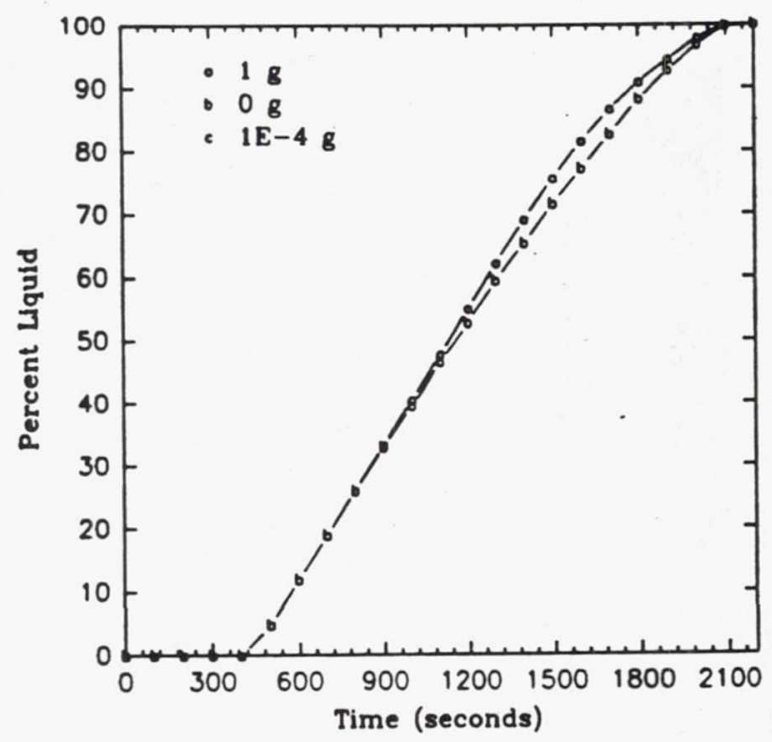

Figure 4 Total Percent Liquid PCM - Melting

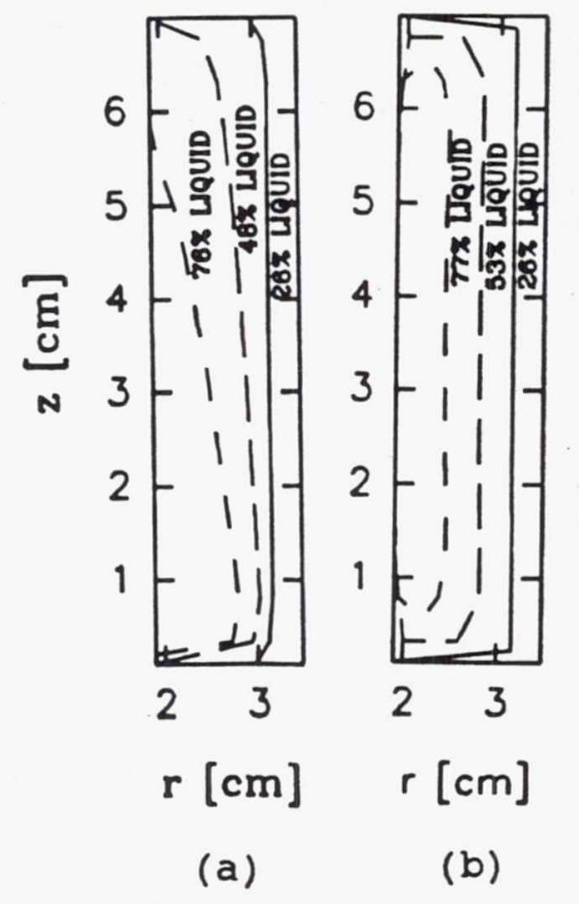

Figure 5 Melting Fronts (a) $1 \mathrm{~g}$ (b) $0 \mathrm{~g} \mathrm{\&} \mathrm{10-4g}$

The PCM melt front surface area is observed to decrease with increasing percent liquid fraction in Figure 5a. The decrease in the liquid/solid interface surface also retards the $1 \mathrm{~g}$ melt (mass) rate as evidenced by the decreasing liquid percent slope in
Figure 4. The decrease in melt front surface area also occurs for t' $\lrcorner \mathrm{e} 0 \mathrm{~g}$ and $10^{-4} \mathrm{~g}$ cases, however, conduction along the top, bottom, and inner walls oppose and slow the decrease in melt front surface area. Therefore, the $1 \mathrm{~g}$ liquid fraction curve in Figure 4 approaches the $0 \mathrm{~g}$ curve near the completion of melting.

The percent liquid versus time for solidification is shown in Figure 6. The $10^{-4} \mathrm{~g}$ and $0 \mathrm{~g}$ curves are again indistinguishable. Solidification for the $1 \mathrm{~g}$ case begins after initiation of solidification for the $0 \mathrm{~g}$ and $10^{-4} \mathrm{~g}$ cases. The fluid mixing due to the buoyancy driven flow delays the onset of melting while the bulk fluid temperature decreases. Therefore a greater amount of sensible heat removal occurs before latent heat is removed for the $1 \mathrm{~g}$ case with respect to the $0 \mathrm{~g}$ and $10^{-4} \mathrm{~g}$ simulations. An additional effect of the $1 \mathrm{~g}$ convection was that more sensible heat was actually removed from the canister wall relative to the $0 \mathrm{~g}$ and $10^{-4} \mathrm{~g}$ simulations. At the completion of solidification, the outer canister wall temperature for the $1 \mathrm{~g}$ case is $4 \mathrm{~K}$ colder than the other two cases. Subsequently, the removal of additional sensible heat during the solidification process resulted in a longer solidification time for the $1 \mathrm{~g}$ case. Freezing times were 1061s and $1055 \mathrm{~s}$ for the $1 \mathrm{~g}$ and $0 \mathrm{~g}\left(10^{-4} \mathrm{~g}\right)$ cases, respectively.

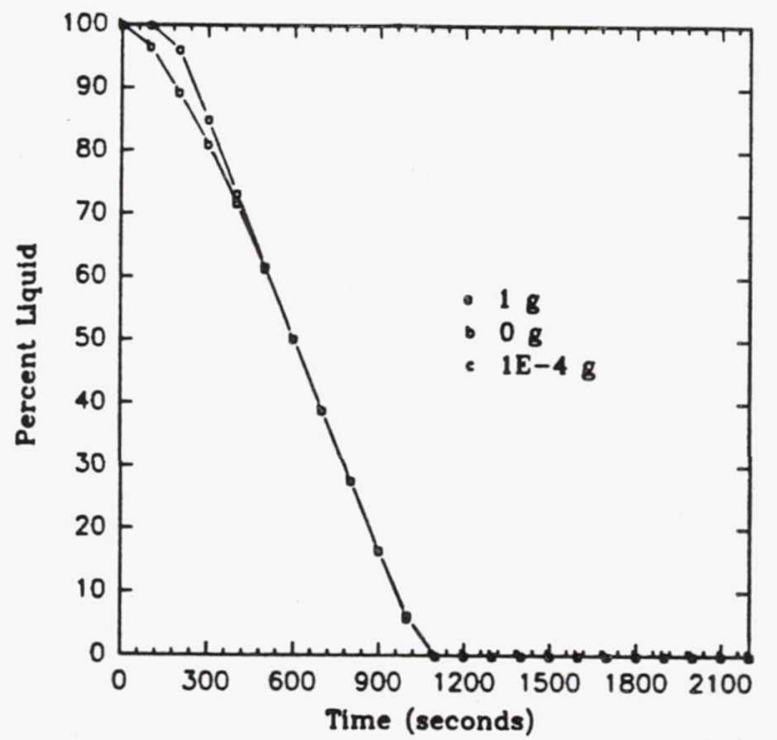

Figure 6 Total Percent Liquid PCM - Solidification

Figure 7a shows the progress of the solidification front for the $1 \mathrm{~g}$ case, while Figure $7 \mathrm{~b}$ shows the progress of the solidification front for the $0 \mathrm{~g}$ and $10^{-4} \mathrm{~g}$ cases. 
Similar to the melting results, the $10^{-4} \mathrm{~g}$ and $0 \mathrm{~g}$ solidification fronts in Figure $7 \mathrm{~b}$ are essentially identical. The greatest front location difference between the $0 \mathrm{~g}$ and $10^{-4} \mathrm{~g}$ cases was $0.004 \mathrm{~cm}$, which was small compared with the PCM thickness $(\Delta r)$ of $1.58 \mathrm{~cm}$. Figure $7 \mathrm{~b}$ reveals that conduction along the top and bottom canister walls also influences the solid/liquid interface shape. A line of symmetry for the solidification front occurs in the radial direction at $\mathrm{z}^{*}=0.5$. For the $1 \mathrm{~g}$ case shown in Figure $7 \mathrm{a}$, the PCM solidified faster near the bottom of the canister than near the top.

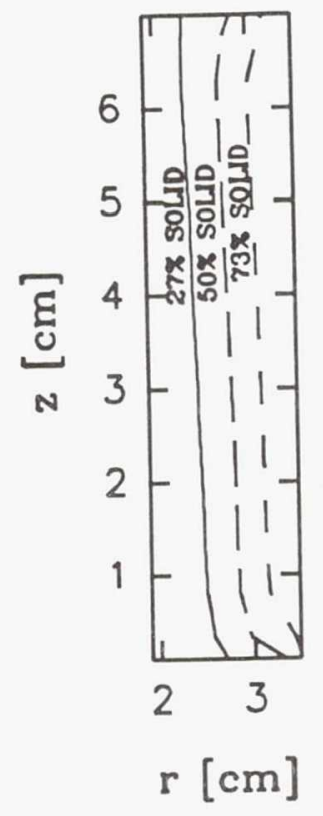

(a)

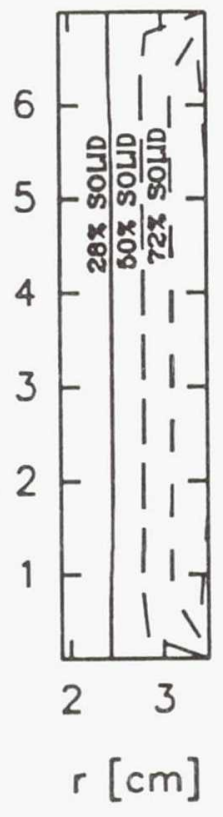

(b)
Figure 7 Solidification Fronts (a) $1 \mathrm{~g}$ (b) $0 \mathrm{~g} \& 10-4 \mathrm{~g}$

The small differences in the temperatures, melting/freezing times, and melting/freezing front profiles for the $10^{-4} \mathrm{~g}$ and $0 \mathrm{~g}$ cases support the similarity analysis conclusion that buoyancy are negligible in a $10^{-4} \mathrm{~g}$ environment for the given TES configuration.

\section{Thermal Energy Storage Flight Experiment}

No microgravity data exist to validate NORVEX. The TES flight experiment will operate in a nominal $10^{-4} \mathrm{~g}$ environment which was shown from similarity and scaling to be a suitable microgravity environment. Therefore the TES flight experiment results will be used to validate NORVEX. Results from ground testing will also be used for validation purposes. The flight experiment and application of the test data to the computer model evaluation are discussed below.

The TES Flight Experiment, $\# 1^{1}$, which consists of an annular geometry containing $\mathrm{LiF}$, is shown in Figure 8. The PCM canister, the conductor rod, and the radiator flare are fabricated from Haynes-188 stock. The canister and conductor rod are an integral piece while the radiator flare, is bolted to the conductor rod. The actual canister assembly is shown in the Figure 9 photograph. Heat flux to the canister is supplied by a cylindrical heating element that radiates heat across a small annular gap to the canister surface. The test package is completely enclosed with multilayer insulation (MLI) to minimize heat losses from the heater and the outer canister wall. This TES assembly shown in Figures 8 and 9 is designed for ground testing; however the configuration will be identical with the flight design.

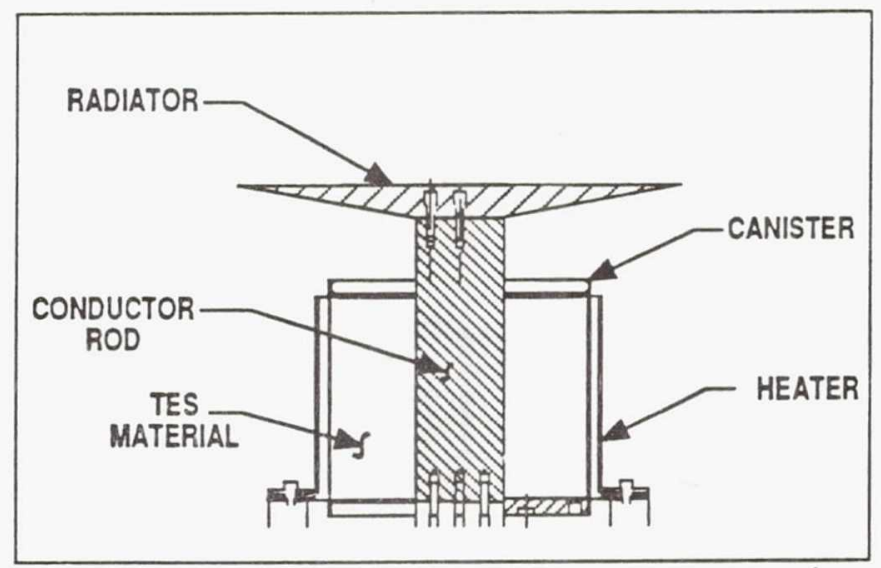

Figure 8 TES Flight Experimental Configuration

Heating continues until the PCM is completely melted at which time heater power is shut off. Simultaneous with the heater cutoff, the shutter above the radiator opens to expose the flared radiator to the heat sink. Cooling occurs as heat is transferred from the PCM through the conductor rod, to the radiator, and to the heat sink. The PCM is subject to a series of melt/freeze cycles approximately equal to the durations of sun and shade in near-earth orbit.

The primary data obtained from the experiment for comparison with simulated results are in the form of temperatures and x-ray (tomography) images. Tomography of the TES canister will be performed after the final PCM solidification. Void size and location can be determined from the tomography 
images. The more fundamental question of whether a single void or multiple voids are formed can also be addressed. Figure 10 is a cross-sectional view of $\mathrm{LiF}$ within a Haynes- 188 canister. The void pattern is clearly delineated as a dark patch in Figure 10. Crosssectional views as the one shown in Figure 10 can be built up to provide a 3-dimensional image of the PCM including void shape.

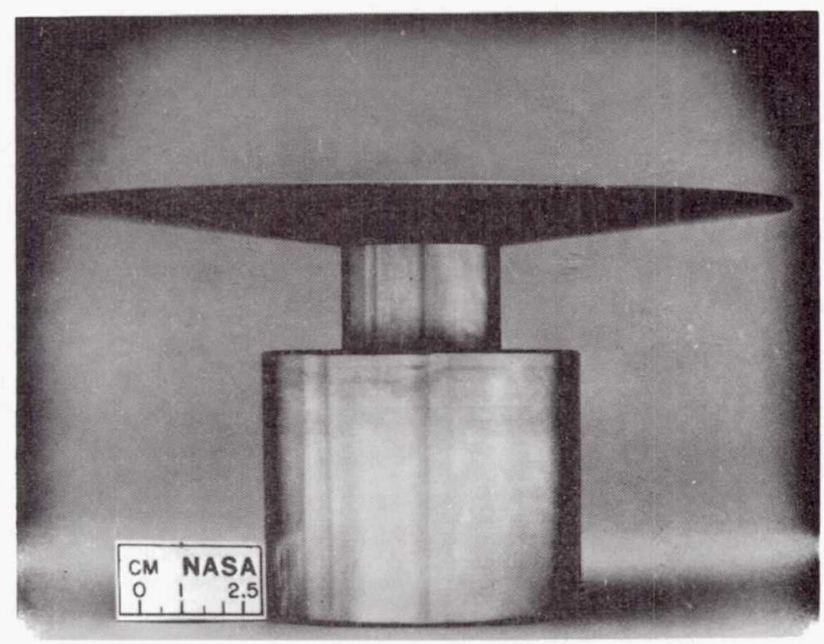

Figure 9 Actual TES Canister Assembly

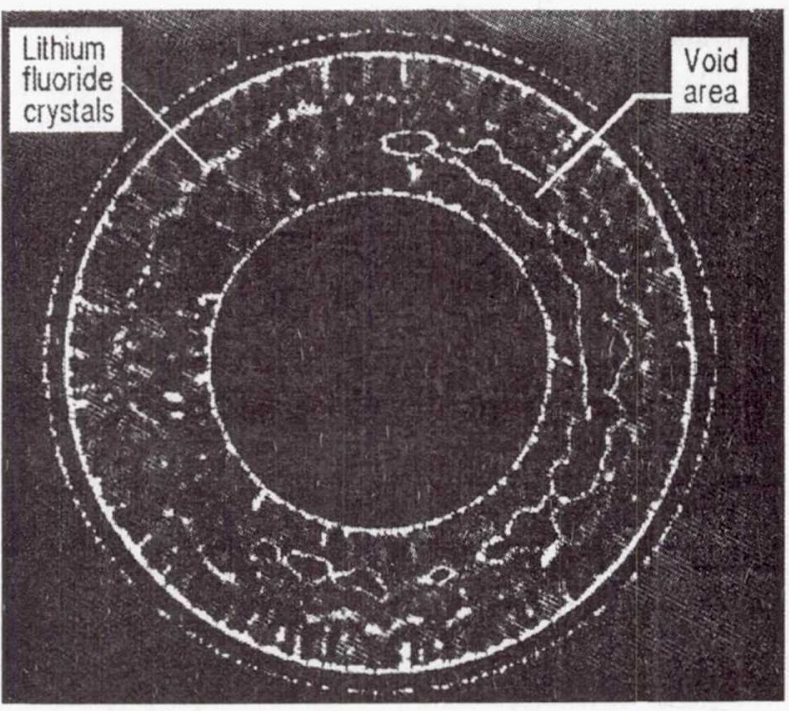

Figure 10 Tomography Image of TES Canister \& PCM

Comparison of the experimental results with NORVEX predictions will be performed in two ways. First, the transient temperature measurements of the canister, initial PCM temperature measurements, and initial canister temperature measurements will be used as inputs to NORVEX. The transient canister temperature measurements are therefore used as time varying boundary temperatures that will drive both the thermal and fluid behavior of the PCM. The final void location, shape, and size from the simulation will be compared with tomography images as shown in Figure 10. The second method for comparing experimental and predicted results will be to use measured heater power, radiator sink temperature, and boundary heat losses as inputs. In this case, the canister temperatures are predicted values in addition to final void location, shape and size that can then be compared to the experimental results. The latter approach is a more rigorous comparison of predicted to experimental results than the former approach. However, the former approach provides greater certainty since less experimental error enters into the comparison. The use of both approaches when comparing the numerical and experimental results will ensure a credible evaluation of the NORVEX computer program.

\section{$\underline{\text { Conclusions }}$}

The following conclusions can be drawn from the scaling analysis and inspection of the relevant dimensionless groups. In general, the free surface boundary conditions and buoyancy terms must be included if modeling of the $1 \mathrm{~g}$ and $10^{-4} \mathrm{~g}$ environments is intended. However, depending on the dominant mechanism, certain terms in the governing equation could be neglected (switched off) during a given simulation to decrease cpu time. In a $1 \mathrm{~g}$ environment, the buoyancy driven flow is expected to remain laminar. In a surface tension driven flow, a boundary layer type flow develops as the PCM melts. Therefore a creeping flow assumption is not valid and the convection terms must be included in the fluid dynamic model. For Lithium Fluoride, a $10^{-4} \mathrm{~g}$ environment is an adequate "microgravity" environment for surface tension to be the dominant flow driving force.

NORVEX was developed to model the PCM behavior during melting and solidification. NORVEX uses porosity terms in the momentum equations to allow the solid, liquid, and mushy PCM to be modeled using a single computational domain. By calculating the gradient of the fluid fraction, the surface tension forces (tangential and normal) can be applied to the cells containing the free surface, and in the correct direction. Data from the TES flight experiment including void location and shape will be used to verify 
the NORVEX model.

Results from NORVEX support the conclusions from the similarity analysis. In a $10^{-4} \mathrm{~g}$ environment and in the absence of free surfaces, buoyancy forces had a negligible effect on the location and movement of the phase change front. The effect of $1 \mathrm{~g}$ convection on total melting or solidification time was small with respect to $0 \mathrm{~g}$ phase change results.

\section{$\underline{\text { References }}$}

1. D. Namkoong "Flight Experiment of Thermal Energy Storage," NASA Technical Memorandum 102081, 1989.

2. T.W. Kerslake, and M.B. Ibrahim, "Analysis of Thermal Energy Storage Material With Change Of Phase Volumetric Effects," NASA Technical Memorandum 102457, 1990.

3. H.J. Strumpf and M.G. Coombs, "Solar Receiver Experiment For The Hybrid Space Station Brayton Engine," Proceedings of the Eleventh Annual ASME Solar Energy Conference, April, 1980.

4. Stefan Weingartner, Jurgen Blumenberg, "Experimental and Theoretical Analysis of Heat of Fusion Storage For Solar Dynamic Space Power Systems," Proceedings of 25th IECEC August, 1990.

5. Kotaro Tanaka, Yoshyuki Abe, Katsuhiko Kanari, Osami Nomura, \& Masayuki Kamimoto, "Advanced Concepts for Latent Thermal Energy Storage for Solar Dynamic Receivers," Space Power, Vol. 8, No.4, 1989.

6. F.P. Incropera \& D.P. Dewitt, Fundamentals of Heat and Mass Transfer, 2nd Ed, John Wiley \& Sons, New York, 1985.

7. A. Bejan, Convection Heat Transfer, John Wiley \& Sons, New York, 1984.

8. J.B. Drake, "Modeling Convective Marangoni Flows with Void Movement in the Presence of Solid-Liquid Phase Change," ORNL-6516, January 1990.

9. FIDAP General Information Manual, Rev 4.0,
Fluid Dynamics Intl, Evanston, IL, September 1987.

10. S. Ostrach, "Low Gravity Fluid Flow," Annual Review of Fluid Mechanics, 14:313-345, 1982.

11. Grodzka and Bannister "Natural Convection In Low-g Environment," AIAA No. 74-156, 1974

12. P.T. Williams, "Thermal Radiative Transport Through LiF For Temperatures Near The Melt Point," ORNL-K/CSD/TM-77, June 1988.

13. R.P. Wichner, "Program NORVEX (NASA Oak Ridge Void Experiment) - NORVEX Users Manual", Oak Ridge National Laboratory, Oak Ridge, TN, 1987.

14. C.W. Hirt \& B.D. Nichols, "Volume of Fluid (VOF) Method for the Dynamics of Free Boundaries," Journal of Computational Physics, 39:201-205, 1981.

15. J.R. Skarda, "Thermal Modeling With Solid/Liquid Phase Change Of The Thermal Energy Storage Experiment," NASA Technical Memorandum 103770, 1991. 


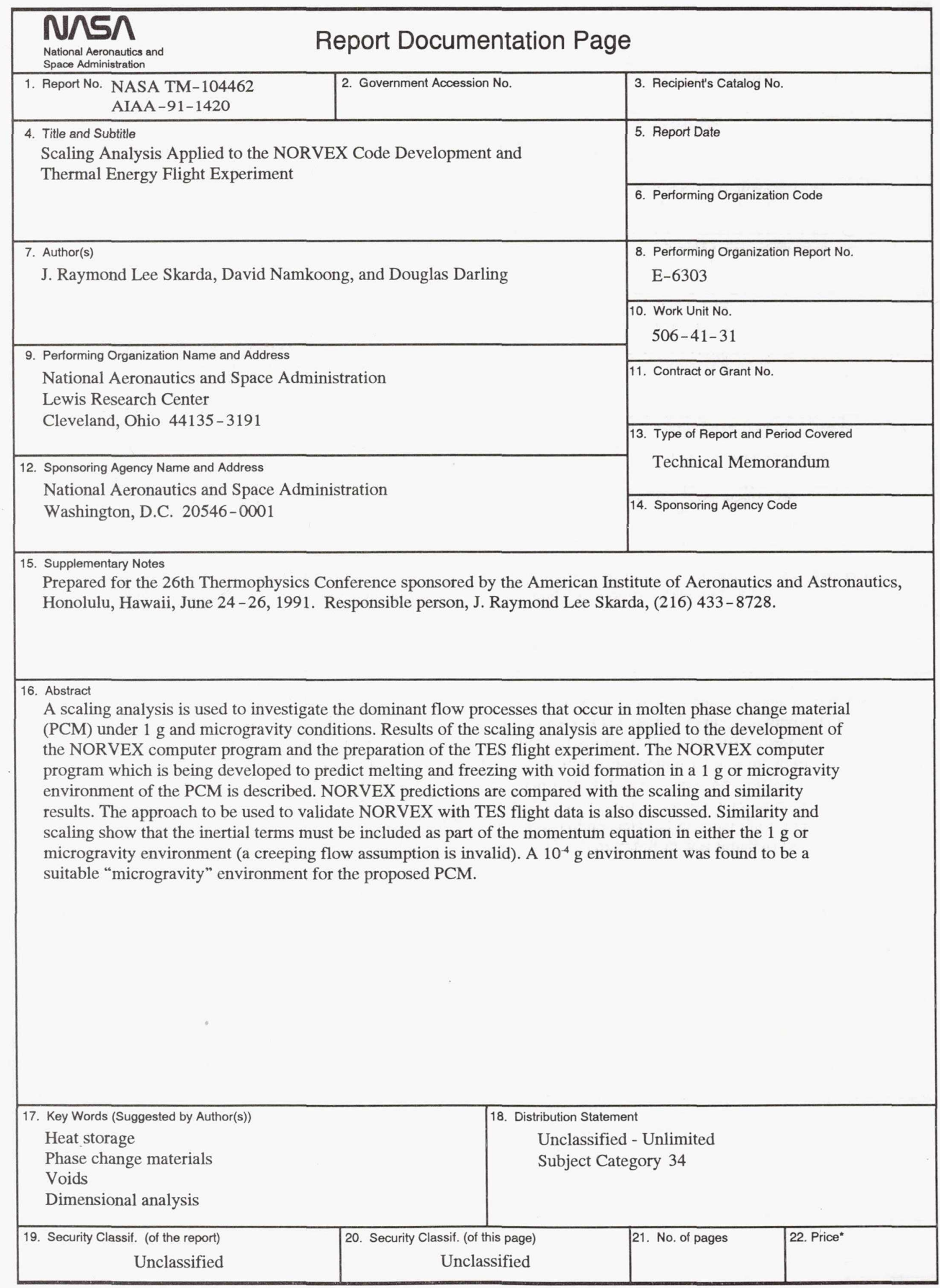

